\title{
Missing teeth after maxillofacial trauma: a case report and management protocol
}

\author{
Ramaraj P.N., Mahabaleshwara C.H., Rohit Singh, Abhijith George, Vijayalakshmi G. \\ Department of Oral and Maxillofacial Surgery, K.V.G. Dental College and Hospital, Sullia, India
}

\begin{abstract}
J Korean Assoc Oral Maxillofac Surg 2020;46:422-427)
Management of maxillofacial trauma includes primary care, in which diagnosis and management of dentoalveolar injury play a vital role. Due to the impact sustained during a maxillofacial injury (whether direct or indirect), dentoalveolar injuries can occur, leading to fracture and displacement of teeth and associated alveolar bone into the surrounding soft tissues and associated structures, such as the maxillary sinus, nasal cavity, upper respiratory tract, tracheobronchial tree, or gastrointestinal tract. Undiagnosed displaced teeth may cause complications such as airway obstruction. This paper reports a case of displaced teeth in the nasal cavity and gastrointestinal tract and highlights the management protocol for displaced teeth secondary to maxillofacial trauma.
\end{abstract}

Key words: Maxillofacial injuries, Tooth injuries, Tooth avulsion

[paper submitted 2018. 9. 26 / revised 2018. 12. 7 / accepted 2018. 12. 16]

\section{Introduction}

Trauma to the oral region occurs frequently and accounts for $5 \%$ of all injuries for which people seek dental treatment ${ }^{1}$. The highest incidence of dentoalveolar trauma occurs in the 20- to 40-year age group ${ }^{2}$. Among all facial traumas analysed in one report ${ }^{2}$, the incidence of dental injuries with respect to the total number of facial injuries was as follows: $57.8 \%$ in play and household accidents, $50.1 \%$ in sports accidents, $38.6 \%$ in accidents at work, $35.8 \%$ in acts of violence, $34.2 \%$ in traffic accidents, and $31 \%$ in unspecified accidents. The overall incidence revealed was $48.25 \%$. Dentoalveolar injury either in isolation or associated with other facial fracture such as zygomatic bone fracture or LeFort I fracture is prone to avulsion and displacement of teeth in adjacent structures, such as the soft tissue, maxillary antrum, nasal cavity, and re-

\section{Rohit Singh}

Department of Oral and Maxillofacial Surgery, K.V.G. Dental College and Hospital, Kurunjibhag, Sullia, Karnataka 574327, India

TEL: +91-8618350707

E-mail:drlomfs@gmail.com

ORCID: https://orcid.org/0000-0003-4997-4415

(c) This is an open-access article distributed under the terms of the Creative Commons Attribution Non-Commercial License (http://creativecommons.org/ licenses/by-nc/4.0/), which permits unrestricted non-commercial use, distribution, and reproduction in any medium, provided the original work is properly cited.

Copyright (C) 2020 The Korean Association of Oral and Maxillofacial Surgeons. All rights reserved. spiratory tract. Such displaced teeth can be accidentally swallowed or spit out by the patient ${ }^{3}$. The following cases about traumatic dislocation of teeth have been reported. One case involved displacement of the mandibular lateral incisor into the nasal floor after panfacial fractures. Diagnostic computed tomography $(\mathrm{CT})$ of the brain and nasal endoscopy were carried out to locate and retrieve the tooth, respectively ${ }^{4}$. Another case reported ingestion of two teeth, one reaching the bronchus and the other the liver, confirmed using fibre-optic bronchoscopy and CT of the abdomen ${ }^{5}$. A final case was of a patient who accidental swallowed a hypodermic needle during root canal treatment and that was removed safely by gastrointestinal endoscopy ${ }^{6}$.

\section{Case Report}

A 26-year-old male reported to the emergency department with chief complaints of laceration and pain in his face. The patient reported a fall from height off a lorry, during which he hit his face on the corner of the lorry and sustained injuries in the orofacial region. Clinical examination revealed bilateral epistaxis, a dentoalveolar fracture with a left zygomaticomaxillary complex fracture, and missing \#11, \#12, \#21, \#22, and \#23 teeth.(Fig. 1) The patient reported that he spat out two teeth at the site of the incident. 


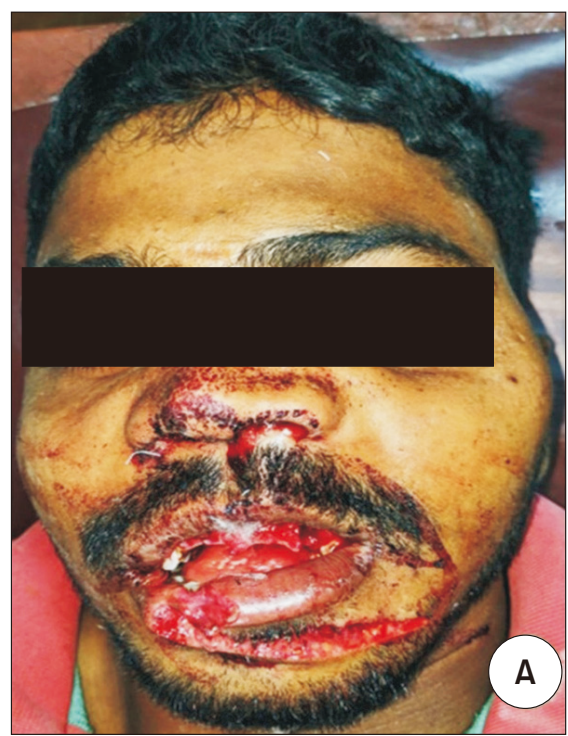

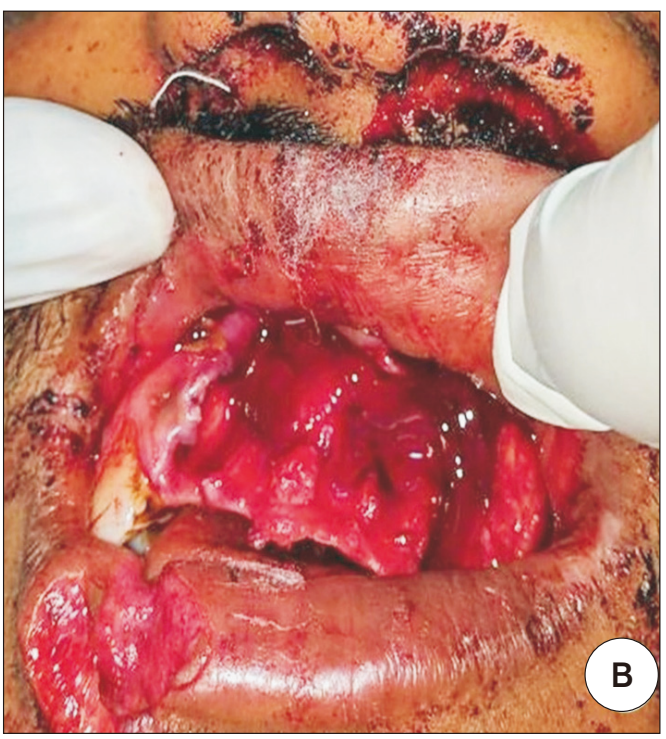

Fig. 1. Preoperative photographs of the patient. A. Frontal view. B. Dentoalveolar fracture with respect to maxillary anterior segments.

Ramaraj P.N. et al: Missing teeth after maxillofacial trauma: a case report and management protocol. $J$ Korean Assoc Oral Maxillofac Surg 2020
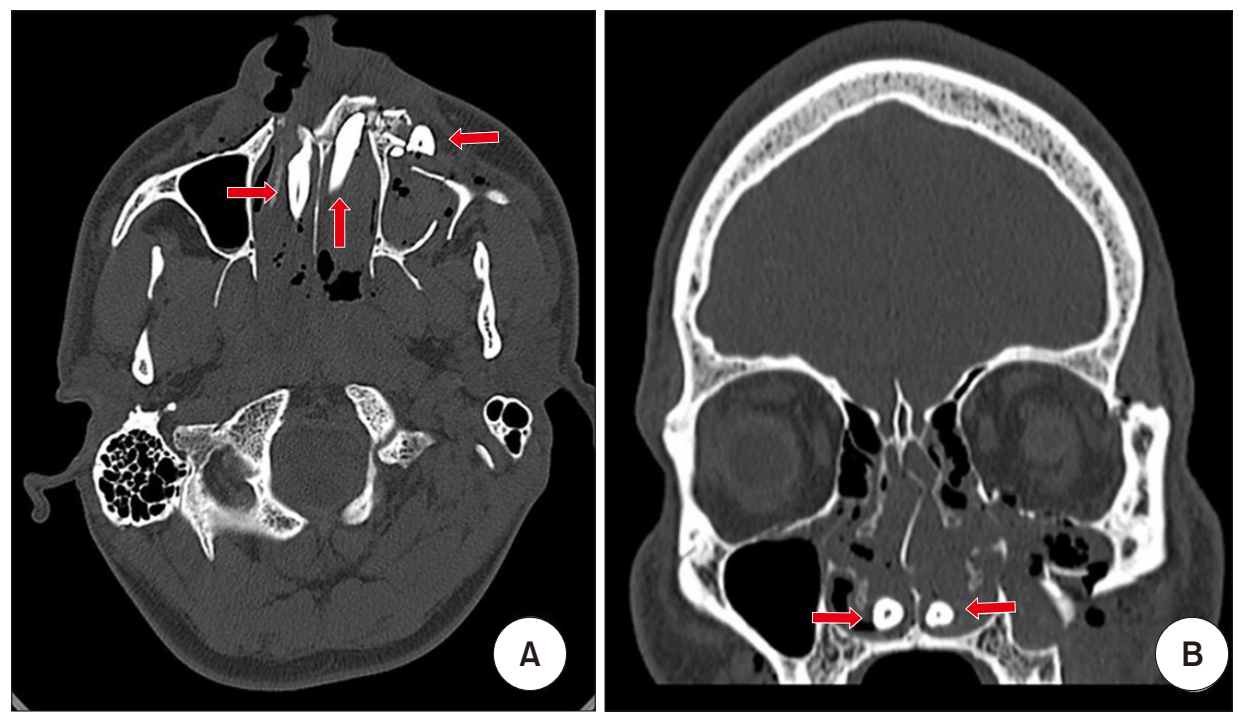

Fig. 2. Computed tomography of the head. A. Axial view: Note the arrows indicating teeth in the nasal cavity and in the soft tissue near the left maxillary sinus. B. Coronal view: Note the arrows showing teeth in the floor of the nasal cavity.

Ramaraj P.N. et al: Missing teeth after maxillofacial trauma: a case report and management protocol. $J$ Korean Assoc Oral Maxillofac Surg 2020

Plain CT scan of the brain revealed multiple dislocations of teeth \#11 and \#21 (Fig. 2) to the respective nasal cavity, and tooth \#23 (Fig. 2. A) intruded completely above the socket and was displaced into the soft tissue near the left maxillary antrum. A lateral neck radiograph, anteroposterior (AP) chest radiograph (Fig. 3), and erect abdominal radiograph were collected to investigate the location of the other missing teeth, but did not reveal any abnormal findings or avulsed teeth.

The patient received primary management with anterior nasal packing for the bilateral epistaxis, and the left maxillary canine, which was displaced in the soft tissue near the left maxillary sinus, was removed using artery forceps.

Open reduction and internal fixation of the zygomaticomaxillary complex fracture and endoscopic removal of teeth from the nasal cavity were planned. On the day of surgery, the patient reported loss of another tooth that day. Intraoperative nasal endoscopy (Fig. 4) did not reveal the missing teeth, which had been seen in the nasal cavity on the CT scan obtained in the emergency department. Postoperative repeat lateral neck, AP chest, and erect abdominal radiographs were collected. Although the neck and chest examinations did not reveal any teeth in the upper airway tract or chest, the radiograph of the abdomen revealed hyperdense structures, i.e., teeth, in the bowel.(Fig. 5. A) We hypothesized that, during primary management using anterior nasal packing, the teeth in the nasal cavities were displaced to the upper aero-digestive tract. One tooth was spat out by the patient on the day of the procedure and the other likely was ingested. Therefore, brain $\mathrm{CT}$ was not repeated. Conservative management for the tooth displaced to the bowel was planned using laxatives, 

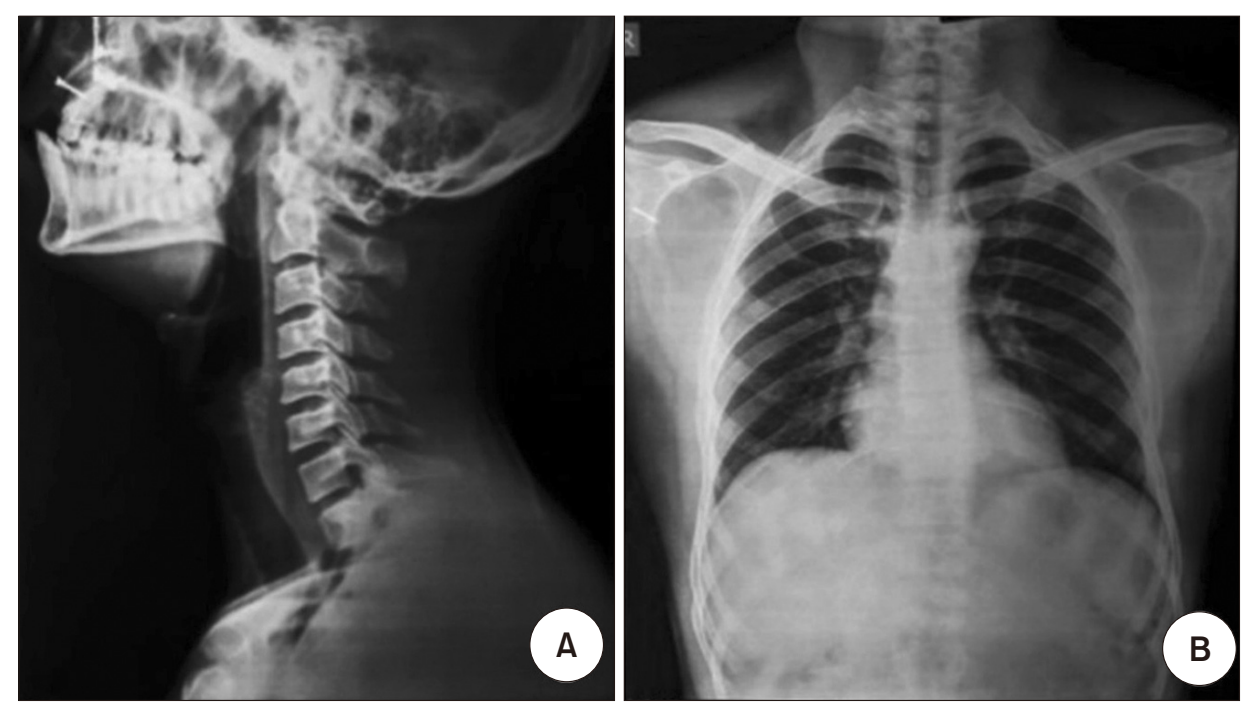

Fig. 3. Radiographs of the tracheobronchial tree and lungs. A. Lateral neck radiograph. B. Anteroposterior chest radiograph.

Ramaraj P.N. et al: Missing teeth after maxillofacial trauma: a case report and management protocol. $J$ Korean Assoc Oral Maxillofac Surg 2020
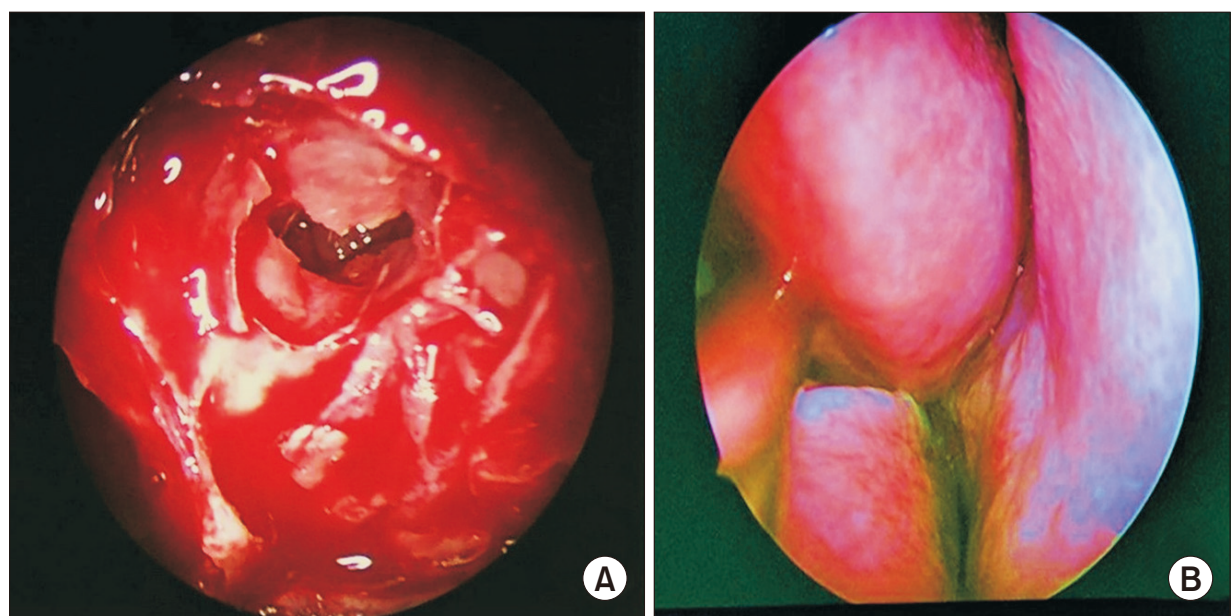

Fig. 4. Nasal endoscopic view. A. The left naris revealed perforation, but no teeth were located. B. The right naris showed no teeth.

Ramaraj P.N. et al: Missing teeth after maxillofacial trauma: a case report and management protocol. $J$ Korean Assoc Oral Maxillofac Surg 2020
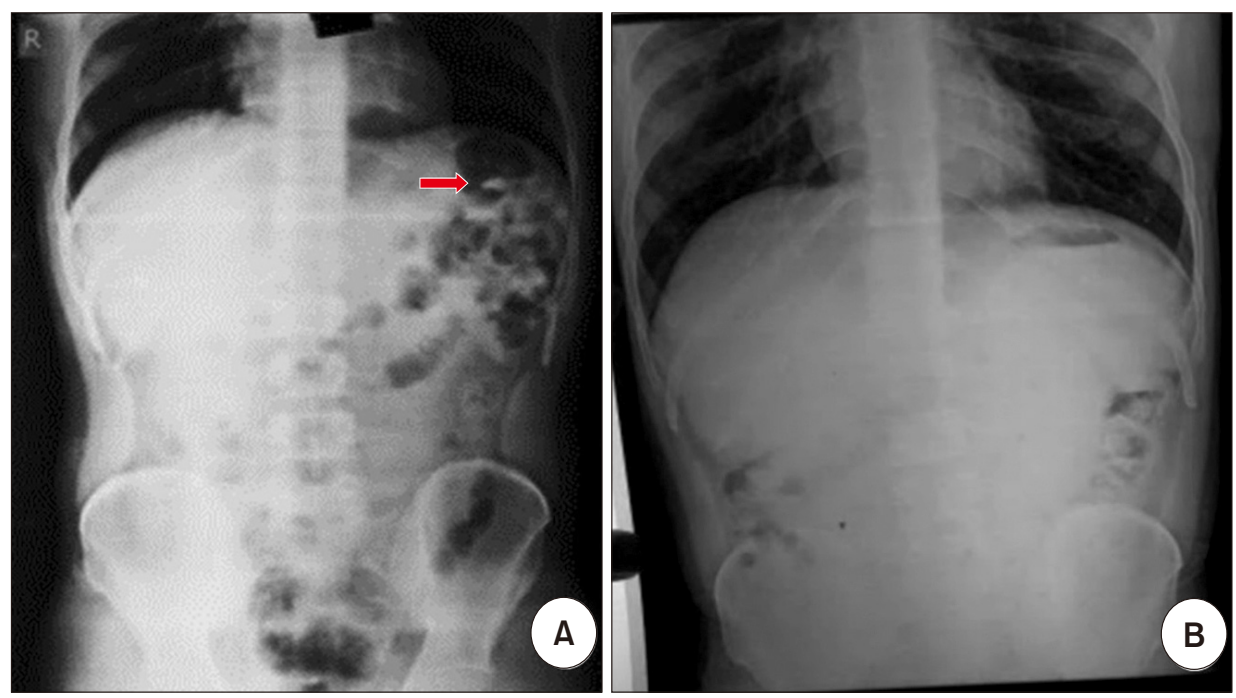

Fig. 5. Radiograph of the gastrointestinal tract. A. Erect abdominal radiograph: Note the arrow showing the hyperdense structure, i.e., a tooth, in the bowel. B. Erect abdominal radiograph: Follow-up radiograph after 7 days of laxative use.

Ramaraj P.N. et al: Missing teeth after maxillofacial trauma: a case report and management protocol. $J$ Korean Assoc Oral Maxillofac Surg 2020 
and the patient was instructed to follow up. After laxative use for seven days, the patient returned for follow up. An erect abdominal radiograph (Fig. 5. B) did not reveal any teeth in the digestive tract. It was concluded that the patient naturally excreted the missing tooth.

\section{Discussion}

Trauma to the oral region occurs frequently and accounts for $5 \%$ of all injuries for which people seek dental treatment ${ }^{1}$.
Among all facial traumas, the overall incidence of dental injuries is $48.25 \%^{2}$. The present case involves midfacial trauma associated with dentoalveolar fracture and displaced teeth in the nasal cavity and digestive tract. An attempt is made to describe a protocol for primary management of midface trauma that includes a dentoalveolar component. Various investigations from basic techniques like X-ray to endoscopic evaluation should be performed to evaluate and manage possible displacement of intruded teeth. Conventional plain radiographs are the first line of investigation in maxillofacial

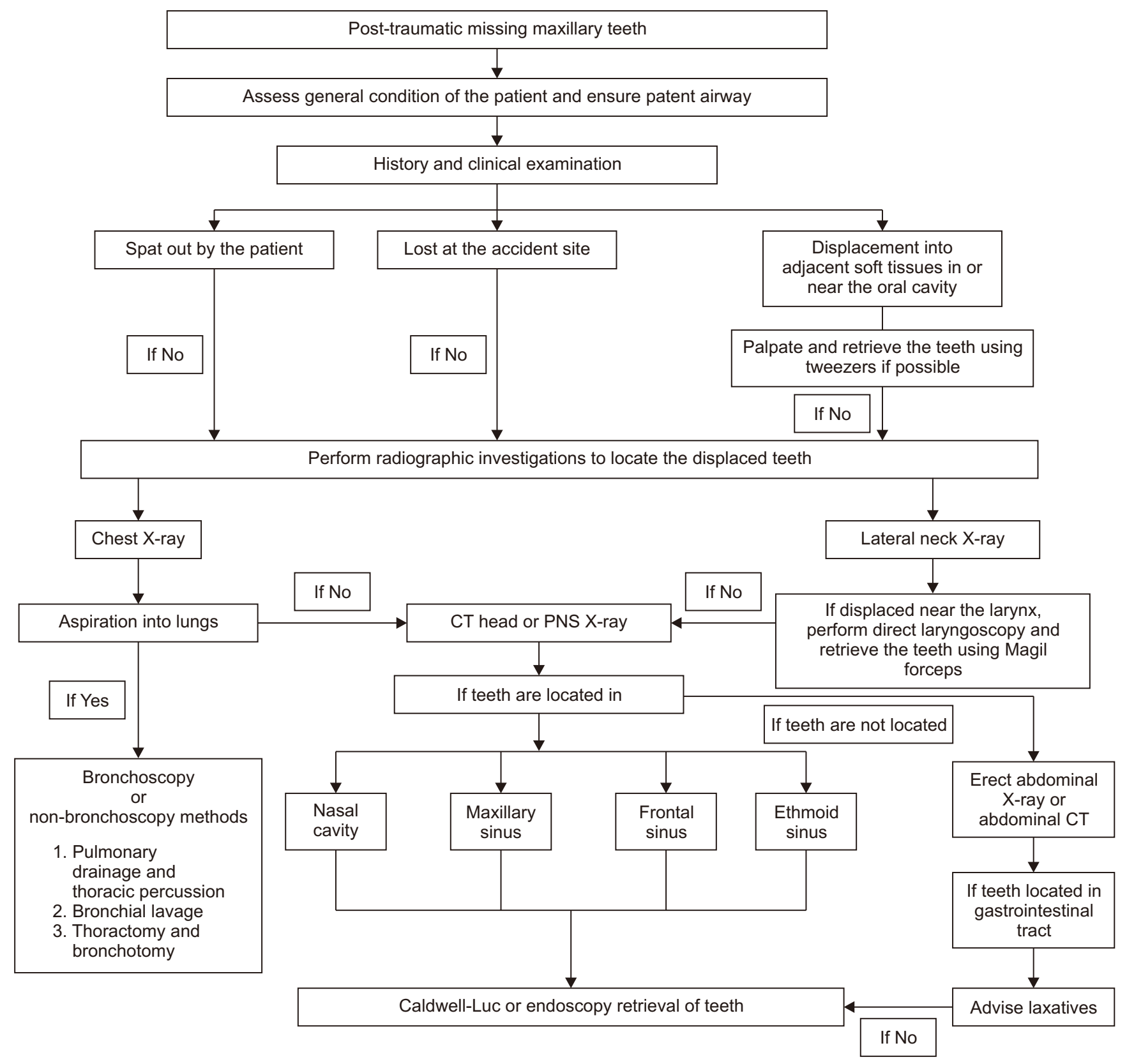

Fig. 6. Protocol for management of displaced missing teeth after maxillofacial trauma. (CT: computed tomography, PNS: paranasal sinus view) Ramaraj P.N. et al: Missing teeth affer maxillofacial trauma: a case report and management protocol. J Korean Assoc Oral Maxillofac Surg 2020 
trauma, but they are limited. Therefore, CT is preferred due to its accuracy of diagnosis.(Fig. 6)

After trauma, intruded teeth missing from the oral cavity or a foreign body can be displaced along three paths: (a) expulsion, (b) aspiration, and (c) ingestion ${ }^{6}$.

In such cases, patient history is very important. In paediatric cases, history of tooth avulsion or expulsion plays a critical role. If the history is not clear, then a hierarchy of clinical examinations and a series of radiographic investigation are vital. Clinical examination involves palpation for missing teeth in the surrounding soft tissue and examination of the oropharynx. If teeth are palpable in the soft tissue near the cavity, every attempt should be made to remove them using artery forceps.

If teeth are displaced in the upper aspect of the trachea or oesophagus, they may be retrieved with simple instrumentation using haemostats, DeBakey forceps, Magill forceps, high-vacuum suction, and/or a laryngoscope ${ }^{7}$.

If the missing teeth are not located, a series of paranasal sinus view, lateral neck, AP chest ${ }^{8}$, and erect abdominal radiographs should be collected. In addition, abdominal CT can be performed. Finally, CT of the head and neck may reveal teeth displaced into the nasal cavity or adjacent paranasal sinuses ${ }^{9}$.

More serious than ingestion, aspiration of teeth must always be treated as an emergency situation. Early complications of aspiration include acute dyspnoea, asphyxia, cardiac arrest, and laryngeal oedema. Thin pointed teeth have higher risk of perforation and pneumothorax than those with a smoother shape.

Delayed removal of a foreign body beyond 24 hours may be associated with increased morbidity and longer hospital stay. Chronic retention of a foreign body can lead to formation of granulation tissue, inflammatory polyps around the foreign body, and obstruction of the bronchus ${ }^{10}$. Aspirated teeth can be removed using either a flexible or rigid bronchoscope; rarely is open thoracotomy needed ${ }^{11}$.

Ilyas et al. ${ }^{5}$ reported a case of ingestion of two teeth, one to the bronchus and the other to the liver. Fibre-optic bronchoscopy revealed a tooth in the posterior segment of the right lower lobe bronchus that was removed by a rigid bronchoscope. Removal of the tooth in the liver was not attempted, as the patient's symptoms were relieved with conservative management.

Foreign bodies that reach the digestive tract usually can be managed with conservative treatment. After 7-10 days, teeth are naturally removed from the digestive tract by the peristaltic movement of the gastro-intestinal tract ${ }^{12}$. In some cases, however, endoscopic removal of teeth is warranted ${ }^{13}$. Jain et al. ${ }^{6}$ reported a case of accidental swallowing of a hypodermic needle by a patient during a root canal treatment, and the needle was safely removed by gastrointestinal endoscopy after 24 hours.

In conclusion, dentoalveolar fracture is common in cases of midface fracture. Teeth may intruded or can avulse and displace into surrounding soft tissues and associated structures, such as the nasal cavity, maxillary sinus, and oropharynx. Avulsed and displaced teeth also can be lost or ingested or aspirated by the patient. Proper history, adequate clinical examination, and a series of radiographs help in locating missing teeth. This paper reported a case of displaced teeth in the gastro-intestinal tract that was managed conservatively, and it provided a brief protocol for diagnosis and management of displaced teeth after maxillofacial trauma.

\section{ORCID}

Ramaraj P.N., https://orcid.org/0000-0003-2526-5673

Mahabaleshwara C.H., https://orcid.org/0000-0003-30299093

Rohit Singh, https://orcid.org/0000-0003-4997-4415

Abhijith George, https://orcid.org/0000-0002-7948-5754

Vijayalakshmi G., https://orcid.org/0000-0002-7922-7472

\section{Authors' Contributions}

R.P.N. and M.C.H. participated in concept and protocol. R.S. and V.G. wrote the manuscript. A.G. participated in collection of data. All author read and approved manuscript.

\section{Consent for Publishing Photographs}

Written informed consent was obtained from the patients for publication of this article and accompanying images.

\section{Conflict of Interest}

No potential conflict of interest relevant to this article was reported.

\section{References}

1. Glendor U. Epidemiology of traumatic dental injuries--a 12 year review of the literature. Dent Traumatol 2008;24:603-11. https:// doi.org/10.1111/j.1600-9657.2008.00696.x

2. Gassner R, Bösch R, Tuli T, Emshoff R. Prevalence of dental 
trauma in 6000 patients with facial injuries: implications for prevention. Oral Surg Oral Med Oral Pathol Oral Radiol Endod 1999;87:27-33. https://doi.org/10.1016/s1079-2104(99)70290-8

3. Ceallaigh PO, Ekanaykaee K, Beirne CJ, Patton DW. Diagnosis and management of common maxillofacial injuries in the emergency department. Part 5: dentoalveolar injuries. Emerg Med J 2007;24:429-30. https://doi.org/10.1136/emj.2006.035949

4. Arunprasad G, Ramasamy A, Madhan B, Krishnan B. Traumatic dislocation of the mandibular lateral incisor into the nasal floor. BMJ Case Rep 2015;2015:bcr2015213553. https://doi.org/10.1136/ bcr-2015-213553

5. Ilyas N, Rajendran VR, Thottath J, Sherinas, Saanida MP, Menon I. Curious odyssey of teeth through body spaces: report of a rare case. Int J Med Res Prof 2017;3:268-70.

6. Jain V, Dubey A, Kumar J, Srivastava S, Tripathy M. Accidental ingestion of a hypodermic needle during root canal treatment: a case report. Gen Dent 2015;63:30-2.

7. Fields RT Jr, Schow SR. Aspiration and ingestion of foreign bodies in oral and maxillofacial surgery: a review of the literature and report of five cases. J Oral Maxillofac Surg 1998;56:1091-8. https:// doi.org/10.1016/s0278-2391(98)90263-4

8. Zerella JT, Dimler M, McGill LC, Pippus KJ. Foreign body aspiration in children: value of radiography and complications of bronchoscopy. J Pediatr Surg 1998;33:1651-4. https://doi.org/10.1016/ s0022-3468(98)90601-7

9. Tung TC, Chen YR, Chen CT, Lin CJ. Full intrusion of a tooth after facial trauma. J Trauma 1997;43:357-9. https://doi. org/10.1097/00005373-199708000-00026

10. Zitzmann NU, Elsasser S, Fried R, Marinello CP. Foreign body ingestion and aspiration. Oral Surg Oral Med Oral Pathol Oral Radiol Endod 1999;88:657-60. https://doi.org/10.1016/s10792104(99)70004-1

11. Kumar N, Goyal H, Bindra A, Goyal K. Management of aspirated tooth in an adult head injury patient: Report of two cases. Saudi J Anaesth 2014;8:276-8. https://doi.org/10.4103/1658-354X.130747

12. Webb WA. Management of foreign bodies of the upper gastrointestinal tract. Gastroenterology 1988;94:204-16. https://doi. org/10.1016/0016-5085(88)90632-4

13. Kuo SC, Chen YL. Accidental swallowing of an endodontic file. Int Endod J 2008;41:617-22. https://doi.org/10.1111/j.13652591.2008.01392.x

How to cite this article: Ramaraj PN, Mahabaleshwara CH, Singh

R, George A, Vijayalakshmi G. Missing teeth after maxillofacial trauma: a case report and management protocol. J Korean Assoc Oral Maxillofac Surg 2020;46:422-427. https://doi.org/10.5125/ jkaoms.2020.46.6.422 